\title{
VENTRICULAR PRESSURE FLOW RELATIONSHIPS IN ISOLATED VALVULAR STENOSIS
}

\author{
BY \\ HAMISH WATSON AND K. G. LOWE \\ From the Department of Medicine, University of St. Andrews, Queen's College, Dundee
}

Received November 20, 1961

The obstruction to the outflow of blood from the right ventricle in pulmonary stenosis has, in the past, been considered largely in terms of pressure gradients recorded between the pulmonary artery and right ventricle during cardiac catheterization and surgical operations, or of the degree of stenosis demonstrated by angiocardiography and autopsy. More recently it has been recognized that muscular hypertrophy is responsible for the infundibular stenosis so often seen in severe cases of pulmonary valvular stenosis, and this has led to more critical appraisal of pressure flow relationships in the right ventricle.

\section{USE OF INTRACARDIAC ELECTROGRAPHY}

Our own early interest in pulmonary stenosis was in the differentiation between valvular and infundibular pressure gradients by means of simultaneous intracardiac electrography and pressure-

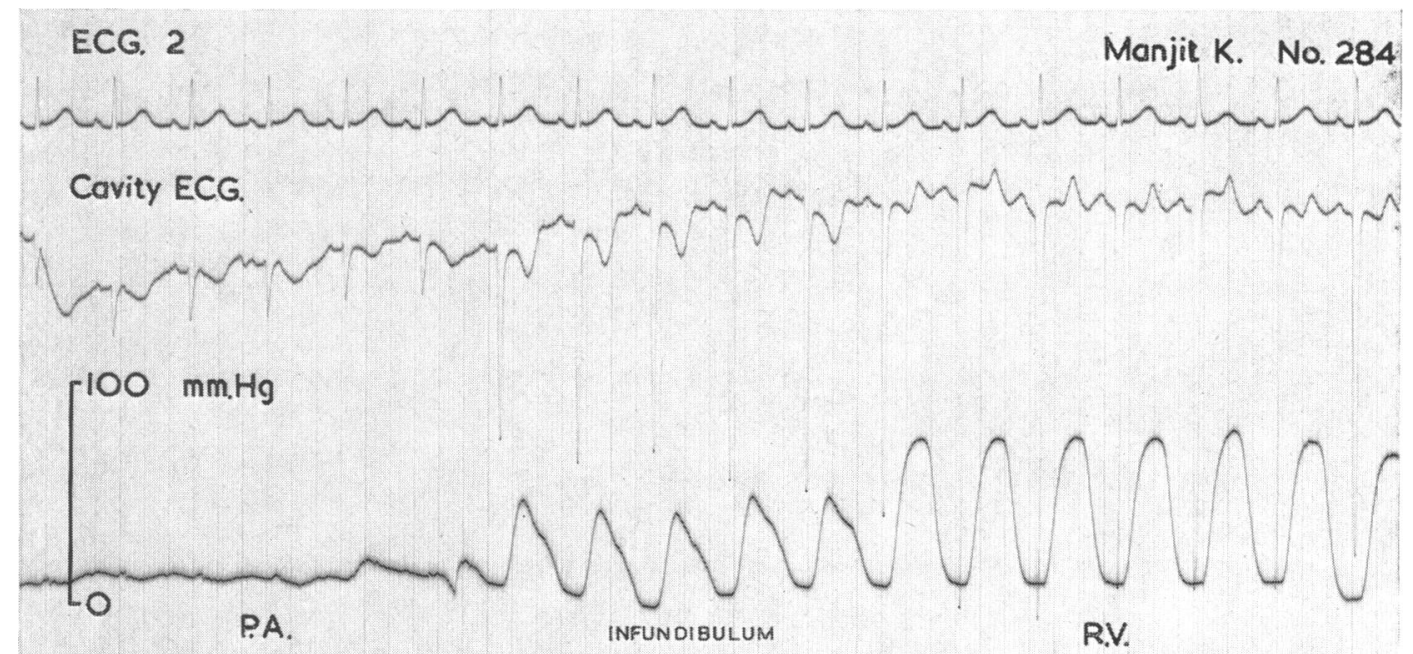

FIG. 1.-A record made as an electrode catheter was withdrawn from pulmonary artery to right ventricle in a patient with Fallot's tetralogy. As the catheter tip passes through the stenosed pulmonary valve there is a systolic pressure gradient and simultaneously a change from pulmonary artery to right ventricular type complexes in the electrocardiogram recorded inside the heart. This marks the site of the valve on the tracing and the fact that the electrocardiogram remains right ventricular throughout the remainder of the recording confirms that the intermediate pressure pulse waves between the two systolic pressure gradients were recorded as the catheter tip passed down through the infundibulum. 
pulse recording (Emslie-Smith et al., 1956). Since then development of this technique has enabled us to recognize and locate with some accuracy the pressure pulses recorded in the outflow tract as a cardiac catheter is withdrawn from the pulmonary artery into the right ventricle. Such a withdrawal tracing is illustrated in Fig. 1 where it will be seen that the electrocardiogram recorded inside the heart changes in pattern from pulmonary arterial to right ventricular type as the electrode at the tip of the catheter passes through the pulmonary valve. This electrocardiographic change is synchronous with the first systolic pressure gradient, indicating valvular stenosis and marking the site of the valve on the record. The intracardiac electrogram remains of right ventricular pattern throughout the remainder of the record and confirms that the pressure pulse waves recorded in the zone of intermediate pressure between the two systolic pressure gradients have been recorded as the catheter tip passed slowly down through the right ventricular outflow tract.

\section{Cine-angiocardiography in Pulmonary Valvular Stenosis}

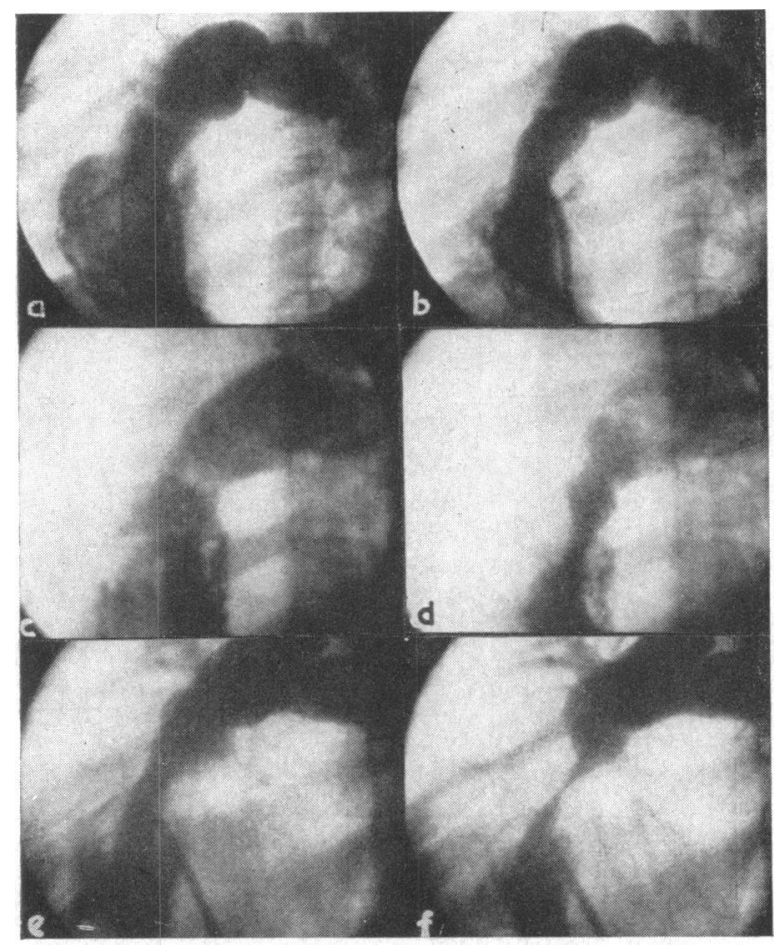

FIG. 2.-Frames selected from cine-angiocardiograms illustrating three right ventricular outflow tracts in cases of mild ( $a$ and $b)$, moderate ( $c$ and $d)$ and severe (e and $f$ ) isolated pulmonary valvular stenosis. This shows the infundibulum fully relaxed during diastole on one side $(a, c$, and $e$ ) and in maximum systolic contraction $(b, d$, and $f$ ) on the other. Note the progressive narrowing of the lumen as the severity of the lesion increases and the extreme narrowing in (f) which is present during most of the cardiac cycle in severe cases.
Later, in the study of the opacified right ventricular outflow tract by conventional angiocardiography, it was found impossible to obtain enough pictures in each phase of the cardiac cycle to distinguish between a normal infundibulum caught in various stages of contraction and relaxation and true infundibular stenosis. This difficulty was overcome by the development of the technique of high speed cine-angiocardiography with image intensification (Watson et al., 1958). With this method the acquired infundibular stenosis caused by muscular hypertrophy in the presence of severe isolated pulmonary valvular stenosis was demonstrated (Watson et al., 1960a), and Fig. 2 shows examples of outflow tracts in cases of mild, moderate, and severe stenosis of the pulmonary valve. It demonstrates the infundibulum fully expanded in diastole on one hand and in maximum systolic contraction on the other, and it will be seen that there is progressive narrowing of the infundibular lumen as the severity of the valvular stenosis increases. In severe cases the infundibulum remains narrow even during its brief phase of relaxation; the bulging hypertrophied muscles encroach on the lumen of the outflow tract throughout its whole length, and from Fig. 2 (f) it is apparent that the right ventricle must encounter great difficulty in emptying its contents through this narrow and almost rigid channel.

\section{Right Ventricular Pressure Pulses in Pulmonary Valvular Stenosis}

Though there now seems no doubt that this secondary hypertrophic infundibular stenosis constitutes an added obstruction to the outflow of blood from the right ventricle (Brock, 1961), the 


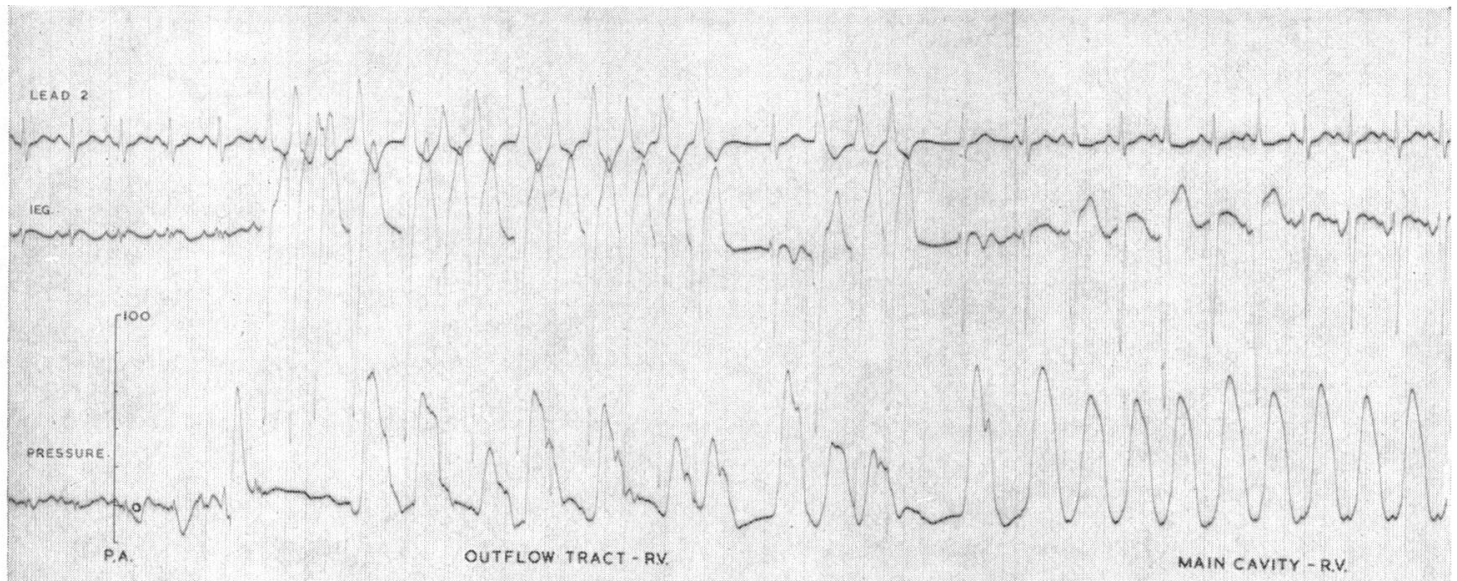

FIG. 3.-Demonstrating that, in pulmonary stenosis, the records in the outflow tract are often spoiled by arrhythmia as the catheter tip stimulates the irritable infundibular muscle during its withdrawal from pulmonary artery into the right ventricular cavity.

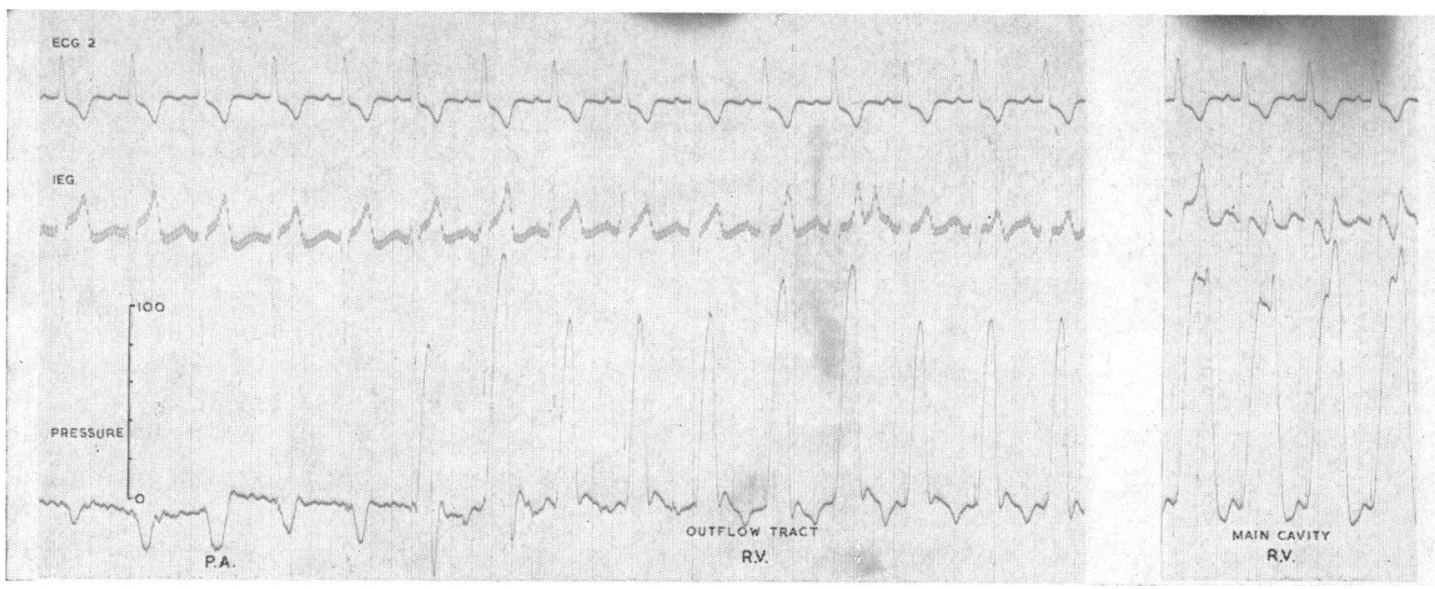

Fig. 4.-Illustrating how in many cases of pulmonary stenosis the difference between the pressure pulses in the outflow tract and the main cavity of the right ventricle may be missed because the recording has been discontinued after the pressure gradient has appeared and the catheter tip is obviously in the right ventricle. The withdrawal tracing is therefore from pulmonary artery to outflow tract only. In this case the systolic pressure pulse recorded in the main cavity of the right ventricle is shown for comparison.

absence of a demonstrated infundibular systolic pressure gradient in such cases has hitherto been somewhat puzzling. There is usually an obvious systolic pressure gradient only at the pulmonary valve, and though the lumen of the whole outflow tract is narrowed, the high pressure seemed to be uniformly distributed throughout the right ventricle.

It is not easy to study the right ventricular outflow tract in pulmonary stenosis on account of its irritability, and usually during cardiac catheterization there are frequent ectopic beats or short bursts of ventricular tachycardia when its walls are stimulated by the catheter tip (Watson, 1962). It is sometimes difficult to pass a catheter even once through the stenosed valve into the pulmonary trunk, and often difficult to do so several times if the records are being spoiled by arrhythmias in 


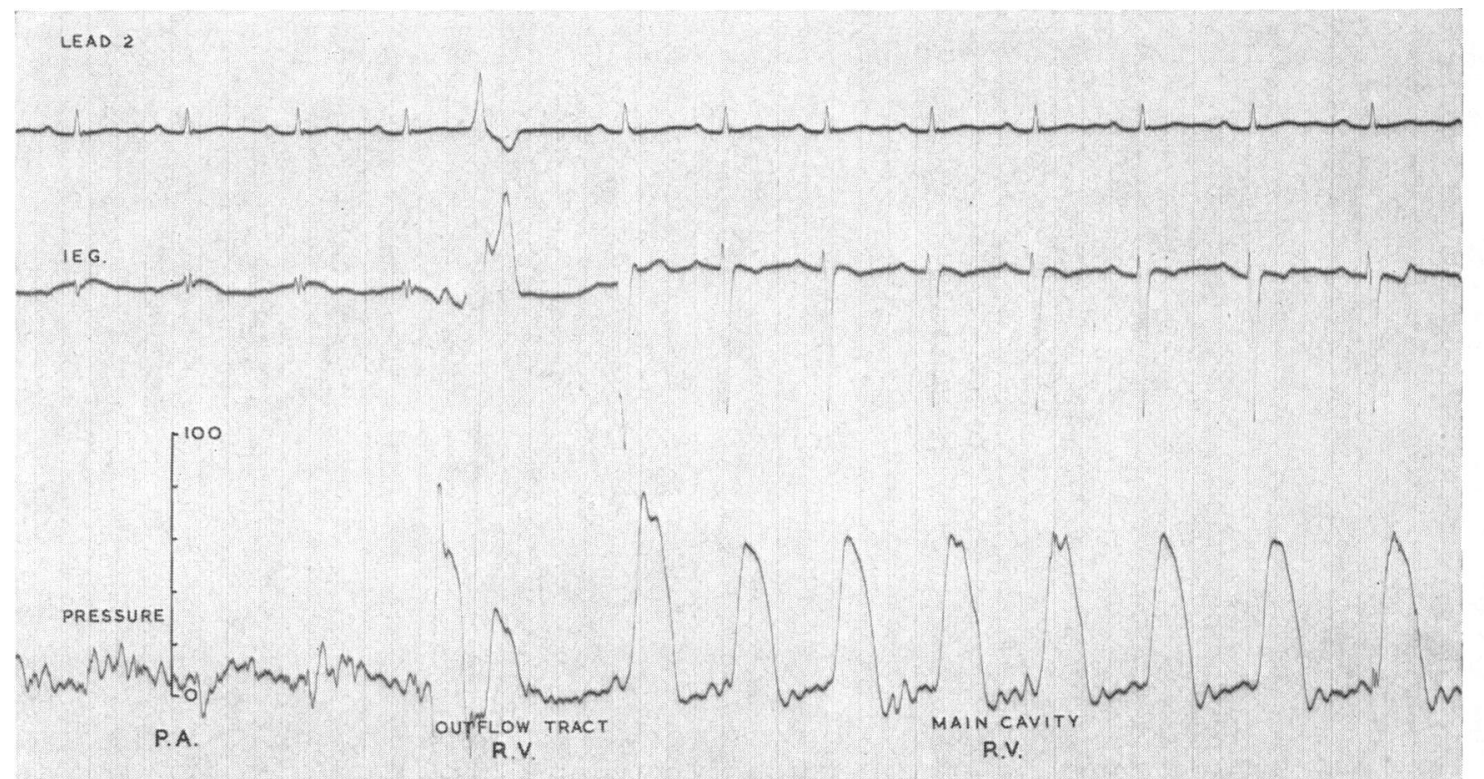

FIG. 5.-A withdrawal tracing in which the catheter tip has passed through the pulmonary valve in systole and thence rapidly through the outflow tract, recording only two ectopic beats on its way from the pulmonary artery to the right ventricle. No true infundibular systolic pressure pulse waves are available for comparison with those recorded in the main cavity.

this way. As a result many withdrawal tracings show at best only one or two genuine right ventricular pressure pulse waves, and the electrocardiograms recorded both from under the pulmonary valve and from the remainder of the infundibulum are bizarre, returning to the usual pattern only when the main cavity of the ventricle is reached. Thus, as will be seen in Fig. 3, the true nature of the pressure and electrocardiographic wave forms in the infundibulum may not be demonstrated. On the other hand, when tracings are not being spoiled in this way by arrhythmias it often happens that only ventricular outflow tract pressure pulses are recorded, because after a systolic pressure gradient has been observed and the pulse waves are obviously right ventricular, withdrawal has been stopped before the main cavity is reached and recording has ended with the catheter tip still lying in the outflow tract. Many published tracings are of this type and Fig. 4 shows the change from pulmonary arterial to infundibular pressure pulses during such a withdrawal and also the rather different type of right ventricular pressure pulse recorded later in mid-cavity.

All those who are familiar with cardiac catheterization know how rapidly a catheter can on occasion change its location inside the heart, whipping from one place to another almost quicker than the eye can follow it. It may thus traverse the outflow tract without recording any true pressure pulses or, at most, only one or two spoilt ones. This is illustrated in Fig. 5 where only two extrasystoles were recorded in the outflow tract during withdrawal of the catheter. On occasion too, the catheter will pass rather rapidly from pulmonary trunk to right atrium with only a few damped or atypical pressure pulses recorded during its whole course through the right ventricle. Fig. 6 shows an example of this, where though the pulmonary stenosis is proved, detailed analysis of the infundibular pressure pulse is not possible. Circumstances may thus combine to make comparison of the infundibular and main cavity pressure pulse waves difficult or impossible and thereby prevent detection of the obvious differences that may exist between them in severe cases of valvular stenosis. 


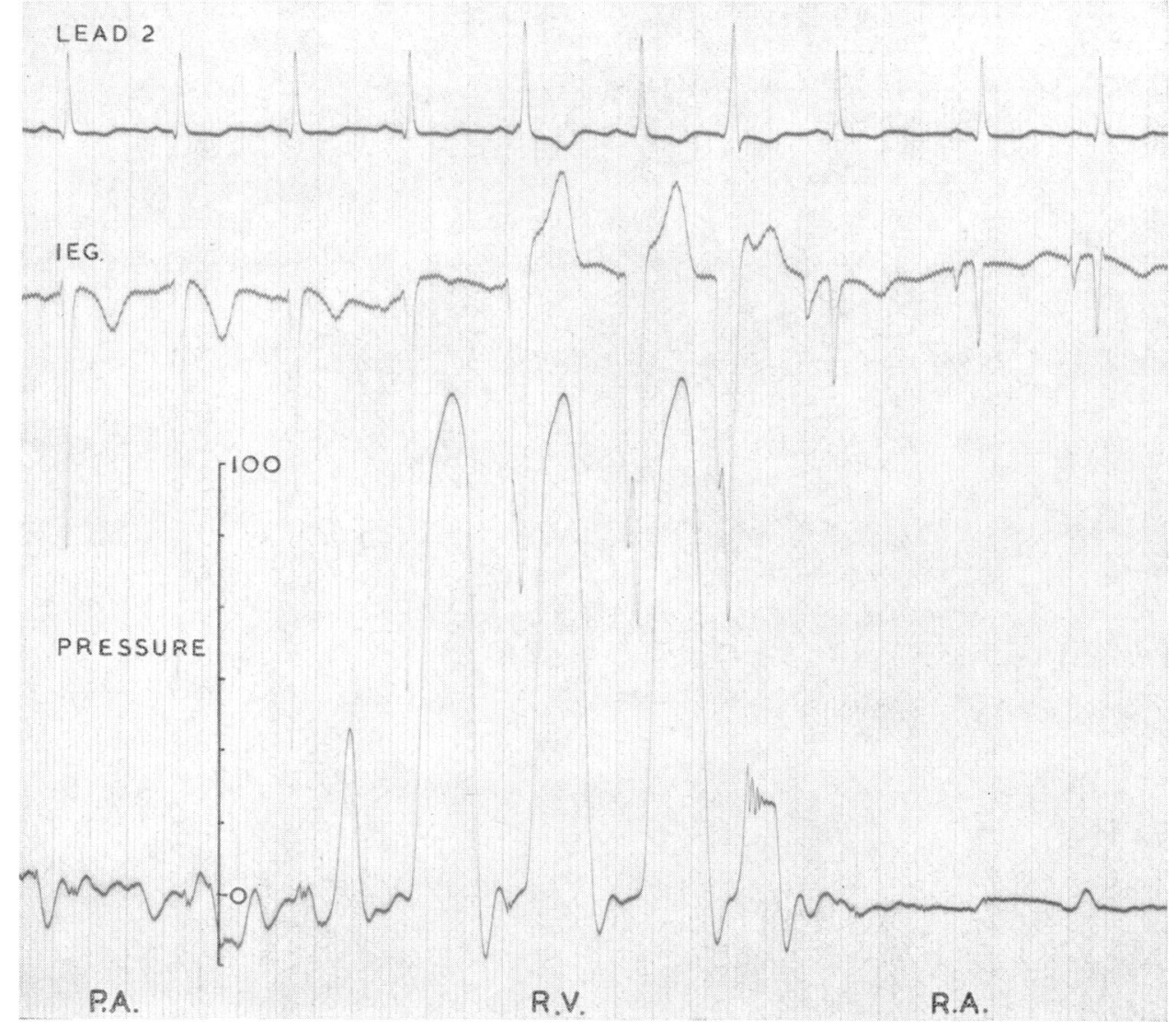

FIG. 6.-On withdrawal the catheter tip has moved very quickly from pulmonary artery to right atrium and though the right ventricular complexes recorded on the way confirm the diagnosis of pulmonary stenosis they are not suitable for detailed analysis.

\section{Pressure Flow Relationships}

Study of pressure flow relationships in man has always been hampered by the fact that most of the physical laws relating to such matters are concerned with steady flow and nearly all are applicable only to rigid tubes. For example Poiseuille's law, $\Delta P=\frac{8 \mathrm{~L} \times V \mu}{\mathrm{R}^{2}}$ where $\mathrm{P}=$ pressure drop, $\mathrm{L}=$ length, $\mathrm{R}=$ radius, $\mathrm{V}=$ volocity, and $\mu=$ viscosity, which deals with streamlined flow of viscous fluids, cannot be applied quantitatively under normal conditions to the circulatory system, because the radius and length of the blood vessels are constantly altering during each cardiac cycle and therefore the pressures and tube dimensions are not independently variable (Rushmer, 1961). We have shown however that the hypertrophied outflow tract in severe pulmonary valvular stenosis appears to be almost rigid and that its radius and length change little once systolic contraction has taken place (Watson et al., 1960b). Though blood is not a truly viscous and homogeneous fluid, this is probably only of importance when considering flow through small vessels, and it seems obvious from Poiseuille's equation that in severe stenosis of the pulmonary valve, where the radius of the outflow tract (R) is greatly decreased and the velocity (V) is therefore greatly increased, we have two factors that will cause the infundibular pressure to fall during systole while the pressure in the main cavity of the right ventricle remains high in order to expel its contents past the obstruction. Even when the whole length of the outflow tract is not uniformly narrowed we know from Bernoulli's theorem, 
$\mathrm{P} / \mathrm{w} \times \mathrm{V}^{2} / 2 \mathrm{~g}=\mathrm{a}$ constant, where $\mathrm{p}=$ pressure, $\mathrm{w}=$ density, $\mathrm{v}=$ velocity, and $\mathrm{g}=$ acceleration due to gravity, that when fluid flows along a rigid tube of varying calibre, the lateral pressure of the fluid at any point varies with the cross-sectional area or inversely with the velocity. The principles of the Venturi pump and flowmeter are, in fact, based on this theorem and the term "Venturi effect" has come to be used in hæmodynamics to describe the drop in pressure that occurs as blood flows rapidly from a high pressure right ventricle through a stenosed pulmonary valve (Sobin et al., 1954; Bouchard and Cornu, 1954). A similar though less marked paradoxical systolic pressure drop has been described in patients with atrial septal defects and greatly increased blood flow through normal pulmonary valves (Jonsson, 1957).

When we considered the hypertrophied muscular infundibulum and the changes in its calibre which had been so clearly demonstrated by cine-angiocardiography, it was obvious that we

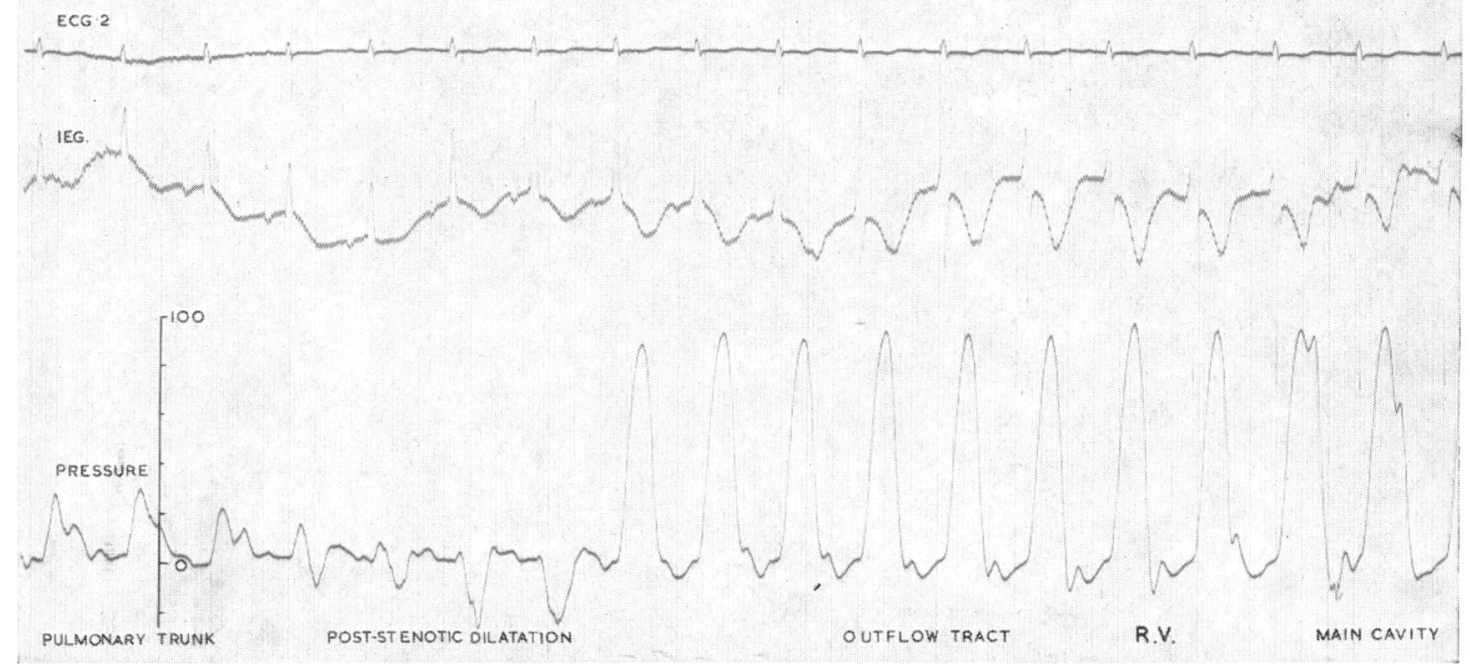

Fig. 7.-A tracing made as an electrode catheter was withdrawn slowly from the pulmonary trunk to main cavity right ventricle. As the tip approaches the pulmonary valve the amplitude of the positive pulmonary arterial pressure pulse waves decreases and the Venturi effect appears. It then slips back through the valve and a large systolic pressure gradient confirms the presence of severe pulmonary valvular stenosis. Its course down through the hypertrophied outflow tract can be clearly followed on the intracardiac electrogram which changes gradually from a tall late $\mathbf{R}$ wave under the valve to the typical rS pattern of mid cavity. It is thus possible to compare the systolic pressure pulse waves: those in the infundibulum are clearly different from the ones recorded in the main cavity of the ventricle (see Fig. 8).

were dealing here with pulsatile flow through an almost rigid tube of complex shape whose lumen was reduced in size during ejection and that the mathematics of the pressure flow relationships must be somewhat complicated. Nevertheless, the fact that the lumen was narrowest when the rate of blood flow was maximum made it clear that a pressure drop was to be expected there during systole. With this in mind we made a fresh appraisal of our cardiac catheterization data in cases of pulmonary stenosis, for though the pulmonary artery Venturi effect is now a well recognized phenomenon, few have appreciated that in pulmonary stenosis there is also a fall in pressure in the right ventricular outflow tract during systole. This is illustrated in Fig. 7 which is a recording made as an electrode catheter was withdrawn slowly from the pulmonary trunk, through a severely stenosed pulmonary valve and thence down a grossly hypertrophied outflow tract into the main right ventricular cavity. As the catheter tip approaches the pulmonary valve the amplitude of the positive pressure pulse waves gradually decreases and the Venturi effect appears, so that the last 
two complexes recorded in the pulmonary artery are negative systolic pressure pulse waves. It then slips back through the valve into the outflow tract and the large systolic pressure gradient confirms the presence of severe pulmonary valvular stenosis. In this case the course of the catheter through the infundibulum can be clearly seen on the tracing because the intracardiac electrogram changes gradually from a high voltage late intracardiac $R$ wave recorded immediately under the valve to the typical rS pattern of mid-cavity. Though there is no obvious systolic pressure gradient present in this case, inspection of the pressure pulse waves shows that those in the outflow tract are not identical

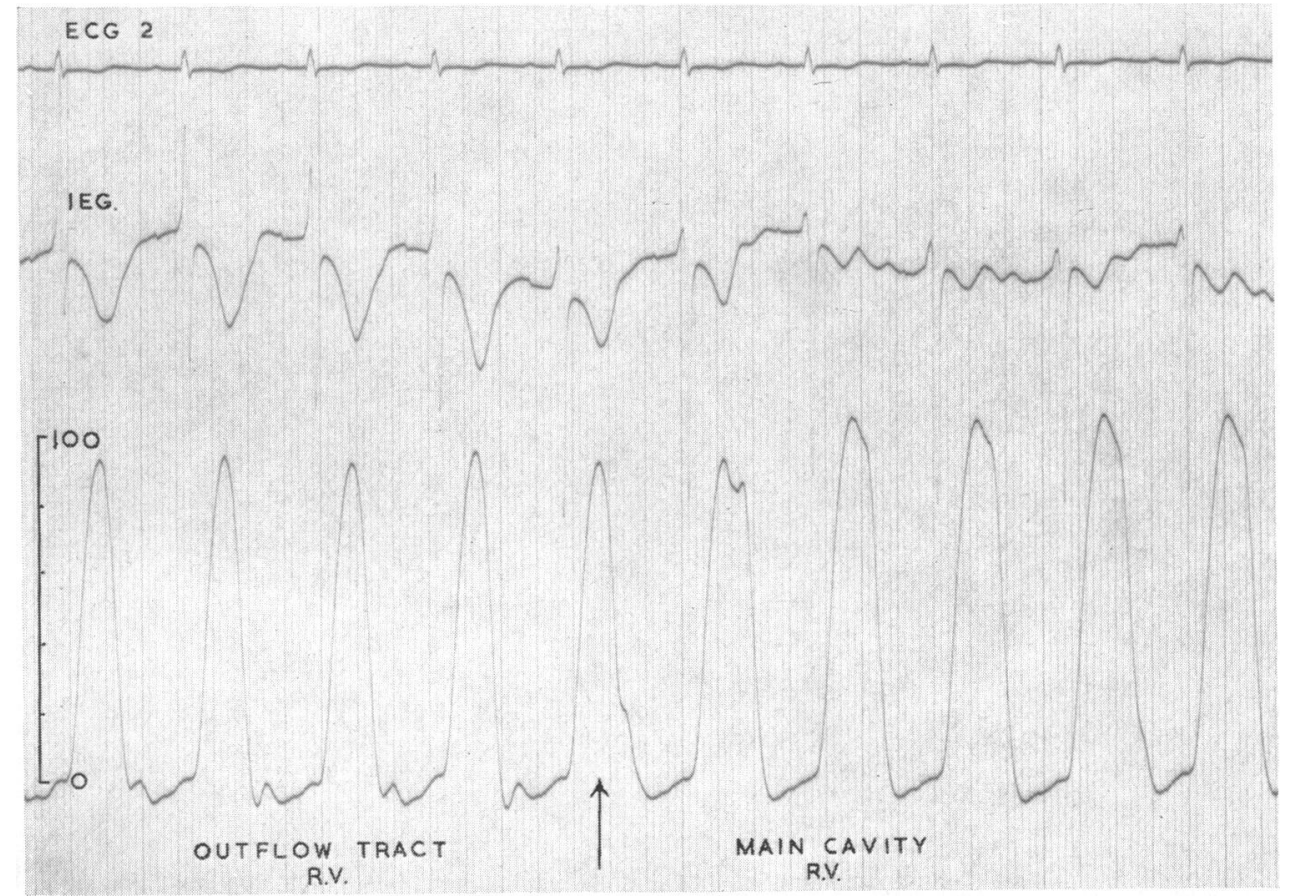

FIG. 8.-An enlargement of the transition from infundibulum to main cavity right ventricle from the case illustrated in Fig. 7. The transitional pressure pulse is marked with an arrow and the two types of right ventricular systolic pressure pulse are seen on either side of it.

with those recorded in the main cavity of the right ventricle. This is seen more clearly in Fig. 8 which is an enlargement of the transition from the infundibulum to the main cavity, and where the transitional pulse is marked by an arrow. The two types of right ventricular pressure pulse can be seen clearly on either side of it. When they are superimposed, using the scalar electrocardiogram to ensure correct timing, Fig. 9A, important differences between them are apparent. Both show the same initial rise in pressure during early systole but then as blood begins to flow at high velocity through the ever narrowing outflow tract the pressure there begins to fall whereas in the main ventricular chamber it remains high throughout systole to force blood past the obstruction. During the remainder of systole the contraction of the hypertrophied infundibular muscles causes further reduction in the lumen of the outflow tract thereby increasing the velocity of the blood flowing through it and consequently decreasing the pressure, which falls away rapidly towards zero. The behaviour of these two pressure pulses is as would be expected by consideration of the physical laws referred to above-narrowing of the bore of the outflow tract causes the blood to flow through it with increased velocity and at decreased pressure. The intraventricular pressure drop is represented by the shaded area in Fig. 9A and similarly the superimposed pulmonary arterial pressure 
pulses in Fig. 9B show the depressor effect that the high velocity jet of blood issuing from the stenosed valve has on the pressure at the valve and in the immediate post-stenotic region. All four pressure pulse waves have been superimposed in Fig. 9C to show their time relationship to each other more clearly.

The pressure flow relationships are therefore broadly speaking as predicted and this fall in systolic pressure in the infundibulum will usually be found when there are true records of both outflow tract and main cavity pulse waves. It may vary at different levels in the infundibulum since its lumen may be conical or irregular in shape, but the sequence of pressure pulses will conform to the general pattern shown in Fig. 10, which is a composite diagram showing the main right ventricle, outflow tract, post-stenotic dilatation, and pulmonary trunk with the corresponding pressure pulse waves.
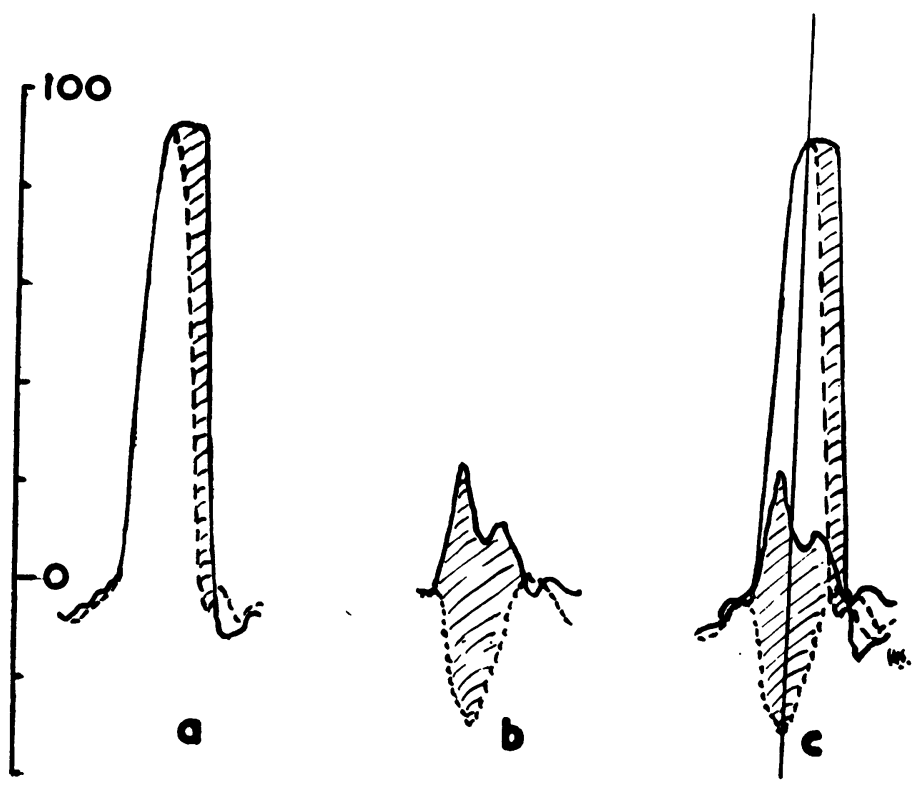

FIG. 9.-In this illustration the systolic pressure pulses from Fig. 7 have been superimposed using the scalar electrocardiogram to ensure correct timing. The differences between those in the outflow tract and main cavity right ventricle due to viscous resistance to flow through the narrow contracting infundibulum are shown in (a) and between those in the pulmonary trunk and the immediate post valvular region because of the Venturi effect in (b). All four have been superimposed in (c) to show their time relationships to each other.

The nature of pressure drops occurring at sites of stenosis during systole depends on the length/ diameter ratio of the obstruction. Thus, where the ratio is small, as at a stenosed pulmonary valve or a localized infundibular obstruction, the fall in pressure is a simple Venturi effect; where it is large in the hypertrophied infundibulum the situation is more complex and depends mainly on viscous resistance to flow through a narrow bore tube. It will be appreciated from Fig. 10 that when flow from the main ventricular cavity begins there will initially be a small Venturi effect as the cross sectional area is suddenly reduced. Then, since flow rate $=$ cross section $\times$ velocity, the blood will flow at high velocity in the narrow bore outflow tract, and as the length/diameter ratio here is high, the fall in pressure along it is mostly a fluid resistance effect. 


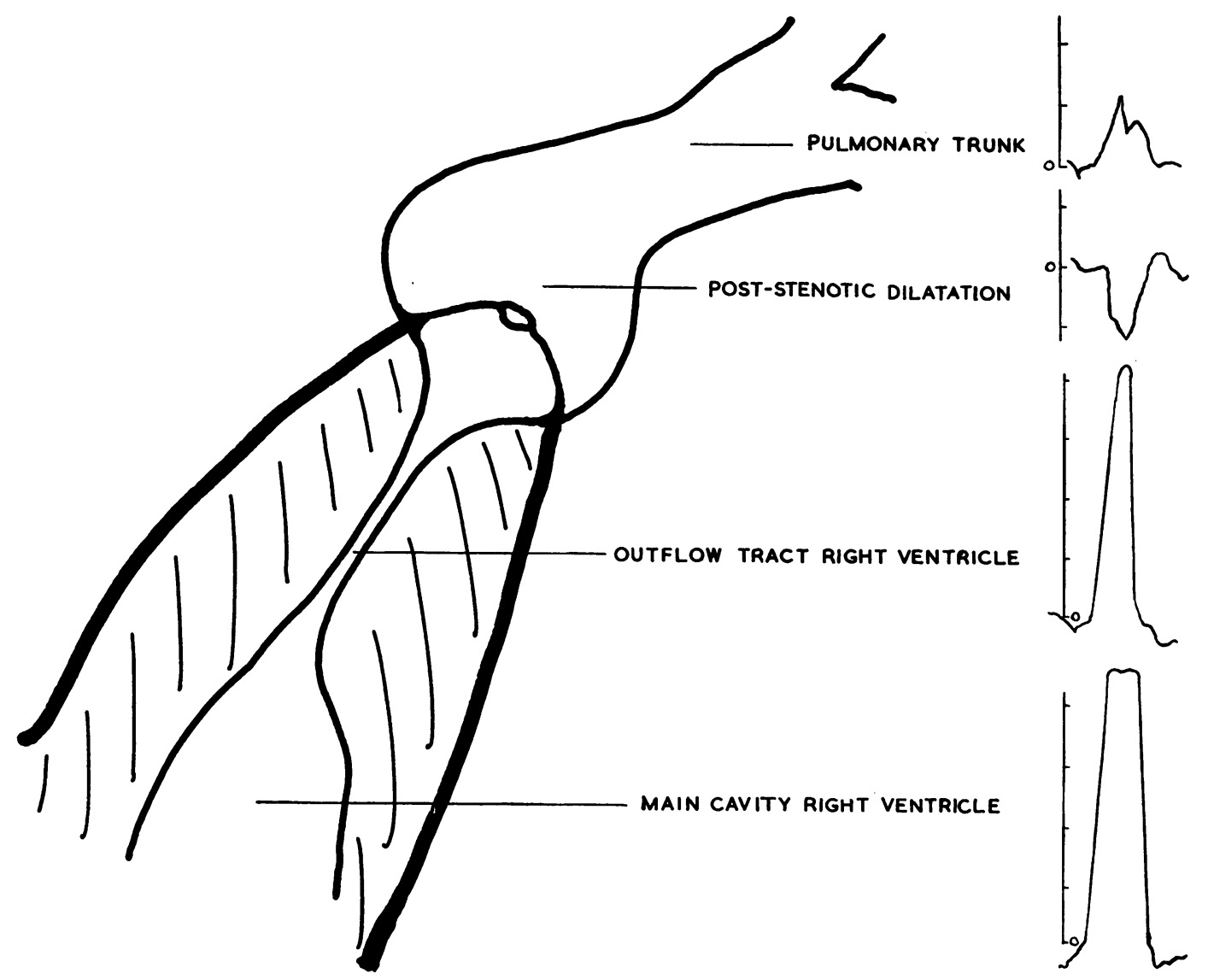

FIG. 10.-A composite diagram showing the different types of systolic pressure pulses that may be found in the right ventricle and pulmonary artery in severe pulmonary valvular stenosis.

\section{Discussion}

There seems little doubt that insufficient attention has been paid to the nature of the wave forms recorded during cardiac catheterization and that unusual patterns have all too often been branded as artefacts of one sort or another. Many abnormal pulses recorded in the infundibulum have, for example, been wrongly attributed to mechanical damping or to movement of the catheter tip backwards through the pulmonary valve in response to the heart's action. This latter seldom, if ever, happens in pulmonary stenosis and though one has indeed to be wary of artefacts when interpreting catheter data they should not be troublesome if a good optical recording system is used and scrupulous attention is paid to technical details.

This interpretation of the nature of the systolic pressure drops seen both at the pulmonary valve and in the outflow of the right ventricle has depended on the study of infundibular pressure pulse waves and the data for this analysis have been collected over a number of years. It has been greatly assisted by the routine use of electrode catheters since these not only locate the site of the tip, but by recording injury currents indicate when it is in contact with or pressed against intracardiac tissues and therefore likely to record damped pulse waves or pressure artefacts. Our better understanding of the hæmodynamics in pulmonary valvular stenosis has therefore been acquired slowly and we were unfortunately unaware until recently of Rodbard's work in this field (Rodbard and Shaffer, 
1956; Rodbard and Rekate, 1957). As they so rightly say "right ventricular and infundibular pressure tracings embodying the features analysed in this study are not uncommon in the literature, but their significance has not been previously commented upon "; they, in their turn were apparently unaware that a similar effect had been illustrated by Kjellberg et al. (1954) and referred to briefly in a case of isolated infundibular stenosis as "the depressor effect of the pressure of velocity." Johnson (1959) has further elucidated the nature of the infundibular pulse waves and we have ourselves, in previous communications, actually reproduced withdrawal tracings that show these characteristic infundibular pressure pulses, though we had failed to recognize them at the time (Emslie-Smith et al., 1956-Fig. 7, complexes 6 and 7; Watson et al., 1960-Fig. 1, complex 5). Rodbard and his colleagues explain these pressure effects phylogenetically with a theory based on delayed activation of the infundibular muscles causing a sphincter like action in mid-systole. Cine-angiocardiographic studies in patients with severe stenosis of the pulmonary valve have made it clear however that the outflow tract is narrow throughout the whole cardiac cycle and that it becomes even narrower during systole.

The realization that muscular hypertrophy is responsible for this infundibular "stenosis" has gone a long way to rationalize the surgical treatment of pulmonary stenosis and even the vexed problem of whether or not to resect the hypertrophied infundibular muscle after successful valvotomy appears to be resolving. As Brock (1961), in his recent review of the subject, has said "nothing continues as simple as it first appears to be, and this has proved to be so with valvotomy for pulmonary stenosis"; we could well echo these words in relation to its diagnosis, for the absence of some sort of pressure gradient between the right ventricle and its outflow tract in such cases has long been a puzzle. This has been solved by a study of the physical laws concerned, and the complex pressure flow relationships that are involved result in a fall in pressure during systole both at the valve and in the outflow tract. Improvements in techniques may yet hold further surprises in store for us and a more careful analysis of the hæmodynamics in other lesions may also be rewarding and increase our understanding of their secondary effects upon the heart.

\section{SUMMARY}

The pressure flow relationships in severe pulmonary valvular stenosis have been studied and a careful examination of the systolic pressure pulse waves recorded during cardiac catheterization shows that they tend to conform to certain physical laws. When the lumen of the right ventricular outflow tract has been narrowed by muscular hypertrophy blood is forced through it with increased velocity and decreased pressure. This fall in pressure during systole produces a characteristic infundibular pressure pulse pattern, which has been illustrated and analysed in some detail and compared with the Venturi effect at the valve.

We would like to record our thanks for the advice given to us during the preparation of this paper by $\mathrm{Mr}$. W. McNicoll and Mr. I. Davidson of the Department of Civil and Mechanical Engineering, Queen's College, Dundee, and to Prof. I. G. W. Hill for his help and encouragement.

\section{REFERENCES}

Bouchard, F., and Cornu, C. (1954). Arch. Mal. Cour, 47, 417.

Brock, R. (1961). Brit. Heart J., 23, 337.

Emslie-Smith, D., Lowe, K. G., and Hill, I. G. W. (1956). Brit. Heart J., 18, 29.

Johnson, A. M. (1959). Guy's Hosp. Rep., 108, 373.

Jonsson, B. J. (1957). Scandinav. J. Clin. Lab. Invest., 9, 249.

Kjellberg, S. R., Mannheimer, E., Rudhe, U., and Jonsson, B. (1954). Diagnosis of Congenital Heart Disease. 1 st ed. The Year Book Publishers, Inc., Chicago.

Rodbard, S., and Shaffer, A. B. (1956). Amer. Heart J., 51, 885.

, and Rekate, A. C. (1957). Exper. Med. Surg., 15, 317.

Rushmer, R. F. (1961). Cardiovascular Dynamics. 2nd ed. W. B. Saunders Company, Philadelphia.

Sobin, S. S., Carson, M. J., Johnson, J. L., and Baker, C. R. (1954). Amer. Heart J., 48, 416.

Watson, H., Pickard, C., Lowe, K. G. and Hill, I. G. W. (1958) Brit. Heart J., $20,459$.

,,,,$+--(1960)$ Brit. Heart J., 22, 706.

, (1962). Brit. Heart J., 24, 144. 